OPEN ACCESS

Edited by:

Wenkai Ren,

South China Agricultural

University, China

Reviewed by:

Zhonghai Yan,

Columbia University, United States

Xiaofei Sun,

University of California, San Francisco,

United States

Denis Roy,

Laval University, Canada

*Correspondence:

Zhiyong Fan

fzyong04@163.com

tThese authors have contributed equally to this work

Specialty section:

This article was submitted to

Nutritional Immunology,

a section of the journal

Frontiers in Immunology

Received: 13 August 2019 Accepted: 14 November 2019

Published: 11 December 2019

Citation:

Ding Y, Song Z, Li H, Chang L, Pan T, Gu X, He X and Fan Z (2019) Honokiol Ameliorates High-Fat-Diet-Induced Obesity of Different Sexes of Mice by Modulating the Composition of the

Gut Microbiota.

Front. Immunol. 10:2800. doi: 10.3389/fimmu.2019.02800

\section{Honokiol Ameliorates}

\section{High-Fat-Diet-Induced Obesity of Different Sexes of Mice by Modulating the Composition of the Gut Microbiota}

\author{
Yanan Ding ${ }^{1,2 \dagger}$, Zehe Song ${ }^{1,2 \dagger}$, Hao $\mathrm{Li}^{1,2}$, Ling Chang ${ }^{1,2}$, Tingli Pan ${ }^{1,2}$, Xueling $\mathrm{Gu}^{1,2}, \mathrm{Xi} \mathrm{He}^{1,2}$ \\ and Zhiyong Fan ${ }^{1,2 *}$ \\ ${ }^{1}$ College of Animal Science and Technology, Hunan Agricultural University, Changsha, China, ${ }^{2}$ Hunan Co-Innovation Center \\ of Animal Production Safety, Hunan Agricultural University, Changsha, China
}

Background: Accumulating data support the fact that the gut microbiota plays an important role in the progression of obesity and its related metabolic disease. Sex-related differences are an important consideration in the study of gut microbiota. Polyphenols can regulate gut microbiota, thereby improving obesity and its associated complications. There have been no studies conducted on the ability of honokiol $(\mathrm{HON}$, an extract from Chinese herbal medicine) to regulate gut microbiota. The aim of this study was to examine whether HON supplementation would improve obesity by regulating the gut microbiota and its related metabolite levels, and whether there were sex-based differences in high-fat diet-induced obese mice.

Methods: C57BL/6 mice $(n=120)$ were fed a normal chow diet (ND group), high-fat diet (HFD group), or HFD plus HON at 200, 400, and 800 mg/kg BW for 8 weeks. Body weight, adipose tissue weight, adipocyte diameter, insulin resistance, blood lipid and serum inflammatory cytokines, gut microbiota, and its metabolite were examined at the end of the experiment.

Results: The HON supplementation reduced body weight, adipose tissue weight, adipocyte diameter, insulin resistance, blood lipid, and serum inflammatory cytokine levels in HFD-fed mice, and this effect was significant in the high-dose group. In addition, HON not only reversed gut disorders in HFD-fed mice, such as by enhanced the abundance of Akkermansia and short-chain fatty acids (SCFAs) producing Bacteroides and reduced Oscillospira, but also improved the SCFAs and endotoxin (LPS) levels, although there were sex-based differences. The correlation between several specific genera and obesity-related indexes was revealed through Spearman's correlation analysis. Moreover, HON may have dose-dependent effects on regulating gut microbiota to alleviate obesity. 


\begin{abstract}
Conclusions: These findings suggest that $\mathrm{HON}$ can prevent diet-induced obesity and its associated diseases by regulating the gut microbiota and improving microbial metabolite levels. Moreover, our findings indicate that sex may be an important factor affecting HON activity.
\end{abstract}

Keywords: honokiol, gut microbiota, metabolites, mice, health

\section{INTRODUCTION}

Obesity and related metabolic diseases, which are caused by a high-fat diet (HFD) and sedentary lifestyle, have been increasing with the improvements in standard of living and the changing lifestyle and have become an urgent public health problem (1). The underlying cause of obesity includes a higher energy storage than consumption due to genetic or environmental factors, as well as an excessive proliferation and differentiation of the body's adipocytes, which ultimately results in weight gain (2). The gut microbiota is made up of trillions of bacteria $(3,4)$ affecting the nutrient digestion and energy metabolism of the host by regulating nutrient uptake and energy and fat storage. It, therefore, plays an important role in the development of obesity and related metabolic diseases $(5,6)$. Studies have found that obese people have a less rich microbiota than lean people (7). High-energy and HFD-induced weight gain and fat accumulation were inhibited in germ-free $(\mathrm{GF})$ mice $(8,9)$. The obese phenotype was also transferred when the microbiota of obese mice was transplanted into GF mice (9). Antibiotic therapy in obese mice reduced obesity and improved glucose metabolism (10). In addition, it was shown that a decrease in Akkermansia and Bacteroides abundance and an increase in Oscillospira abundance are closely related to the occurrence and development of obesity (11-13). These results indicate that microbiota plays an important role in the development of obesity. Moreover, obesity would lead to an increase in serum LPS concentration (14), which is the main component of the outer membrane of Gram-negative bacteria (15). Lipopolysaccharide could lead to metabolic inflammation and insulin resistance (14). Studies have demonstrated that Akkermansia can improve the body's inflammatory response and protect the intestinal barrier (11), Oscillospira is closely related to the body's inflammatory response and intestinal permeability (13), and Bacteroides can promote health by producing short-chain fatty acids (SCFAs) to provide energy for intestinal epithelial cells and inhibit LPS-induced inflammation $(12,16)$. These results suggest that regulating gut microbiota may be an important measure to prevent obesity and obesity-related metabolic syndrome.

There is increasing evidence that plant functional components, especially polyphenols, can regulate gut microbiota, thereby improving obesity and its associated complications $(17,18)$. For example, the addition of apple proanthocyanidins significantly increased the ratio of Bacteroidetes/Firmicutes, and the abundance of Akkermansia, alleviated the weight gain, inflammation, and intestinal permeability, and improved lipid metabolism induced by a HFD (17). Supplementing a basic diet with resveratrol changed the gut microbiota in mice. When this gut microbiota was transplanted into HFD-fed mice, the recipients had less weight gain, increased insulin sensitivity, alleviated inflammation, and improved lipid metabolism and intestinal barrier function (18). In addition, gut microbiota could play a beneficial role in the host by producing SCFAs, such as butyrate, which provide energy for intestinal epithelial cells and inhibit inflammation induced by LPS, promoting health $(12,16)$. These results suggest that polyphenols can improve obesity and related complications by regulating gut microbiota and metabolites.

Honokiol $(\mathrm{HON})$ is a naturally occurring, pleiotropic lignan that was confirmed as one of the main biological active components of Magnolia officinalis, a traditional Chinese medicine (19). HON has been shown to have broad-spectrum antibacterial (20), anti-tumor (21), neuro-modulating (19), and anti-oxidation activities (22) by modern pharmacological tests, and has now become more widely studied due to its pleiotropic effects. In previous reports, supplementation of $\mathrm{HON}$ and magnolol ameliorated body fat accumulation, insulin resistance, and adipose inflammation in HFD-fed mice (23). However, only $5-10 \%$ of $\mathrm{HON}$ are absorbed in the small intestine as dietary polyphenol. The rest are transported to the posterior intestine for microbial degradation and utilization, and shape the structure of gut microbiota (24). Therefore, it cannot be ignored that the role of gut microbiota in the process of HON acting on the host. In addition, studies have confirmed that sex-related differences are an important consideration in the study of gut microbiota. For example, women had fewer metabolic disorders caused by diet $(25,26)$, and inflammatory bowel disease caused by microbiota changes in the gut was more common in women than in men $(27,28)$. However, whether there are sex-related in the regulation of high-fat-induced gut microbiota, improving obesity and related complications by $\mathrm{HON}$, has not been studied.

Therefore, the aim of this study was to investigate whether HON can improve obesity and its related complications by regulating gut microbiota, and whether this effect was associated with sex differences.

\section{MATERIALS AND METHODS}

\section{Materials}

HON samples (purity $>99.9 \%$ ) were purchased from Victory Biological Technology Co., Ltd. (Sichuan, China).

\section{Animal and Experimental Design}

After approval by the Committee on the Ethics of Animal Experiments of Hunan Agriculture University, 120 C57BL/6 mice (SPF, 6 weeks old, 60 male and 60 female) were purchased 
from the Hunan SJA Laboratory Animal Co., Ltd (Hunan, China). All mice were housed $2 /$ cage at $23 \pm 2^{\circ} \mathrm{C}$ with $50 \pm$ $5 \%$ relative humidity and with a 12 -h light-dark cycle. After 1 week of acclimation, the mice were randomly divided into five groups randomly (24 mice per group, half male and half female), and fed a normal chow diet (ND group, D12450J, 10\% kcal from fat, $3.85 \mathrm{kcal} / \mathrm{g}$, Research Diets, Inc., USA), high-fat diet (HFD group, D12492, 60\% kcal from fat, $5.24 \mathrm{kcal} / \mathrm{g}$, Research Diets, Inc., USA), and HFD with HON at $200 \mathrm{mg} / \mathrm{kg} \mathrm{BW} \mathrm{(H200}$ group), $400 \mathrm{mg} / \mathrm{kg} \mathrm{BW} \mathrm{(H400} \mathrm{group),} \mathrm{and} 800 \mathrm{mg} / \mathrm{kg}$ BW (H800 group) for 8 weeks. Body weight was measured weekly, and food intake was recorded daily. After 8 weeks of experimental treatment, blood samples were collected from the orbital plexus and the mice were sacrificed. The adipose tissues were removed, rinsed with a physiological saline solution, weighed, and stored at $-80^{\circ} \mathrm{C}$ until analyzed. Meanwhile, cecal contents were collected in Eppendorf tubes and immediately stored at $-80^{\circ} \mathrm{C}$ for subsequent analysis. The animal experimental protocols were conducted in accordance with the Institutional Animal Care and Use Committee of Hunan Agriculture University.

\section{Insulin Resistance Assessment}

The blood insulin of fasted mice (overnight) was measured using a commercial ELISA kit (Wuhan Cusabio biotech Co., Ltd., China), according to the manufacturer's recommendations. Blood glucose was determined with an automatic biochemical analyzer (Shenzhen Mindray Bio-Medical Electronics Co., Ltd., China). The homeostasis model assessment-insulin resistance (HOMA-IR) was calculated using the following formula: HOMA-IR = fasting blood glucose $(\mathrm{mmol} / \mathrm{L}) \times$ fasting insulin $(\mathrm{mU} / \mathrm{L}) / 22.5$.

\section{Biochemical Analysis and Cytokine Measurements}

Serum triglyceride (TG), total cholesterol (TC), low-density lipoprotein cholesterol (LDL-C), and high-density lipoprotein cholesterol (HDL-C) were determined by an automatic biochemical analyzer (Shenzhen Mindray Bio-Medical Electronics Co., Ltd., China). Free fatty acids (FFA) were determined using a commercial kit (Nanjing Jiancheng Bioengineering Institute, Nanjing, China). Serum LPS-binding protein (LBP), tumor necrosis factor- $\alpha$ (TNF- $\alpha)$, interleukin6 (IL-6), interleukin-1 $\beta$ (IL-1 $\beta$ ), and interferon- $\gamma$ (IFN- $\gamma$ ) concentrations were then quantified using commercial ELISA kits (Wuhan Cusabio biotech Co., Ltd., China).

\section{Histological Examination}

Epididymal WAT was dissected, washed in saline, and immediately fixed in $4 \%$ paraformaldehyde. Fixed tissues were embedded in paraffin and $4-\mu \mathrm{m}$ sections were prepared and stained with hematoxylin and eosin (H\&E) for general morphological observations. Images were acquired at $200 \times$ magnification. The sizes of white adipose tissue was measured using Photoshop CS6 (Adobe Systems Inc., San Jose, CA, USA).

\section{SCFA Analysis}

About $60 \mathrm{~g}$ of cecal contents were weighted accurately using a $1 / 10000$ balance, added to $1.5 \mathrm{ml}$ of ultrapure water, and vortexed uniformly for 2 min overnight. The mixtures were then centrifuged at $12,000 \mathrm{~g}$ for $15 \mathrm{~min}$, and the supernatants and $25 \%$ metaphosphoric acid were mixed in a proportion of $\mathrm{v}: \mathrm{v}$ $=9: 1$ for SCFA analysis. The SCFA content was determined using an Agilent 7890A at a programmed temperature (Agilent Technologies Inc., USA).

\section{Gut Microbiota Analysis}

Five cecal content samples from each group were selected for microbiota 16S rRNA analysis. Total bacterial genomic DNA samples were extracted using a Fast DNA SPIN extraction kit (MP Biomedicals, Santa Ana, CA, USA), following the manufacturer's instructions. The quantity and quality of the extracted DNAs were measured using a NanoDrop ND-1000 spectrophotometer (Thermo Fisher Scientific, Waltham, MA, USA) and agarose gel electrophoresis, respectively. The V3V4 hypervariable region of the bacterial 16S rRNA gene was amplified using universal primers (338F and 806R). Sequencing was performed on the Illumina HiSeq platform using HiSeq2500 PE250 (Illumina, USA).

The Quantitative Insights Into Microbial Ecology (QIIME, v1.8.0) pipeline was used to process the sequence data. Briefly, raw sequencing reads with exact matches to the barcodes were assigned to their respective samples and identified as valid sequences. Low-quality sequences were filtered according to the criteria of Chen and Jiang (29). After chimera detection, the remaining high-quality sequences were clustered into operational taxonomic units (OTUs) at $97 \%$ sequence identity by UCLUST (30). The abundance of each OTU in each sample and the taxonomy of these OTUs are represented by an OTU table. Data analyses were mainly performed using QIIME and R packages (v3.2.0). OTU-level alpha diversity indices, such as the Chao1 richness estimator, ACE metric (Abundance-based Coverage Estimator), Shannon diversity index, and Simpson index, were calculated using the OTU table in QIIME. Beta diversity analysis was used to investigate the structural variation of microbial communities across samples using UniFrac distance metrics (31) and visualized via principal coordinate analysis (PCoA), nonmetric multidimensional scaling (NMDS), and the unweighted pair-group method with arithmetic means (UPGMA) hierarchical clustering (32). Venn diagrams were generated to visualize the shared and unique OTUs among samples or groups using the R package Venn Diagram, based on the occurrence of OTUs across samples/groups regardless of their relative abundance (33). A heatmap was created on the basis of the top 50 dominant genera using $\mathrm{R}$ packages (V3.5.2). The Spearman's rho nonparametric correlations between the gut microbiota and obesity-related indexes were determined using $\mathrm{R}$ packages (V3.5.2).

\section{Statistical Analysis}

Data are expressed as the mean \pm SEM (the standard error of the mean). A two-sided unpaired Student's $t$-test with BenjaminiHochberg correction was used to compare the two groups. The 
differences among the four groups were analyzed using oneway analysis of variance (ANOVA) followed by Duncan's test. Significance was set at $P<0.05$.

\section{RESULTS}

\section{HON Suppresses HFD-Induced Increases in Body Weight of Mice}

In female and male mice, the body weight and weight gain of HFD-fed mice were significantly higher than those of the NDfed and HFD $+800 \mathrm{mg} / \mathrm{kg} \mathrm{HON}$-fed mice at the end of study $(P<0.05$, Figures 1A-C). The HFD $+200 \mathrm{mg} / \mathrm{kg} \mathrm{HON}$ diet had similar effects on male mice $(P<0.05$, Figures $1 \mathrm{~A}-\mathrm{C})$. In addition, while the weights of the female mice in the H800 group significantly decreased compared to those of female mice in the HFD group by 7 weeks, the difference in the weights of the male mice was seen 4 weeks earlier $(P<0.05$, Figures 1A,B). Total food intake did not vary significantly among the HFD-fed groups of female mice and male mice except for the H800 group (Figure 1D), where a significant decrease of food efficiency ratio (FER) was observed for the H800 group of female mice and H800 and H200 groups of male mice compared with the HFD group $(P<0.05$, Figure 1E), suggesting that the effects of HON on body weight did not result from the reduction of food intake but from the FER (weight gain divided by food consumption weight). These results imply that $\mathrm{HON}$ reduced weight gain in HFD-fed mice and that there were sex-based differences in the body weight, weight gain, and FER $(P<0.05$, Figures $1 \mathrm{C}-\mathrm{E})$; Moreover, the food intake of HFD $+800 \mathrm{mg} / \mathrm{kg}$ HON male mice was notably lower than that of the female mice $(P<0.05$, Figure 1D).

\section{HON Prevents Increases in Adipose Tissue Mediated by a HFD in Mice}

Since HON decreases the body weight of HFD-fed mice, we investigated whether the decreased body weight gain was due to a reduction in adipose tissue mass by weighing the fat pads. As shown in Figures 2A,B, the HFD significantly increased weights of the abdominal WAT, epididymal WAT inguinal WAT, perirenal WAT, and mesenteric WAT fat pads over those of the ND-fed group. HON supplementation decreased the HFDinduced gains in WAT weights, especially at high doses $(P<0.05$, Figures 2A,B). Histological sections from the inguinal WAT of HFD group showed that adipocyte diameter was greater in the HFD than the ND group, while adipocyte size was clearly smaller in the H200, H400, and H800 groups than in the HFD group $(P<0.05$, Figures $2 \mathrm{C}-\mathbf{F})$. Adipose mass was correlated with body weight in the mice fed HON, suggesting that the HONinduced decrease in body weight increase could be attributed to a reduction in adipose tissue weights. Results showed that HON significantly decreased adipocyte diameter in inguinal WAT compared to the HFD group $(P<0.05$, Figures $2 \mathbf{C}-\mathbf{F})$, suggesting that $\mathrm{HON}$ may inhibit adipogenesis and hyperplasia of inguinal WAT.

\section{HON Ameliorates Serum Lipid Profile and Attenuates Systematic Inflammation in HFD-Fed Mice}

Obesity is accompanied by increased serum lipid levels and inflammatory cytokine levels (34). As shown in Figure 3, compared to the ND-fed group, mice in the HFD-fed group had significantly increased serum levels of TG, TC, HDL-C, LDL-C, FFA, TNF- $\alpha$, IL- 6 , and IL- $1 \beta$, and decreased IFN- $\gamma$ serum levels. HON supplementation remarkably reduced serum lipid levels (TG, TC, FFA, and LDL-C) and systematic inflammation (TNF$\alpha$ and IL-1 $\beta)$ compared to those in the HFD group $(P<0.05)$. HFD plus HON at 400 and $800 \mathrm{mg} / \mathrm{kg}$ significantly increased serum levels of IL- 6 and decreased FFA concentration $(P<$ 0.05 , Figure 3$)$. In addition, male mice had lower serum levels of IL- 6 and IL- $1 \beta$ than female mice and there was no significant difference between TC and FFA.

\section{HON Reduces Insulin Resistance in HFD-Fed Mice}

Studies indicated that diet-induced obese mice would have significantly increased fasting glucose and an elevated HOMAIR value (34). In the present study, all the HON treatments, except for the high dose of HON on male mice, resulted in a significant reduction in fasting glucose and insulin levels along with a decrease in HOMA-IR in HFD-fed mice $(P<$ 0.05 , Figures $4 \mathbf{A}-\mathbf{C}$ ), suggesting that $\mathrm{HON}$ can improve insulin resistance. In addition, there were sex-related in fasting glucose and HOMA-IR.

\section{Effects of HON Treatment on the Gut Microbiota Composition in Diet-Induced Obese Mice}

Increasing evidence shows that the gut microbiota plays an important role in the development of obesity and obesity-related complications $(6,35)$. Therefore, after 8 weeks of feeding, we sequenced cecal content samples to elucidate the effects of ND, HFD, and HFD supplemented with $\mathrm{HON}$ on the gut microbiota structure. In female mice, at all detected, 672 OTUs were detected in all groups (Figure 5A). There were 248, 181, 100, 127, and 227 unique OTUs in the ND, HFD, H200, H400, and H800 groups, respectively. There was no significant difference in the value of ACE, Chao1, Shannon, and Simpson between the ND, HFD, H200, and H400 groups (Table S1). However, significant differences in ACE and Chaol were found between mice in the HFD and H800 groups (Table S1). In male mice, 559 OTUs were presented in all groups (Figure 5B). There were 256, 147, 100,192 , and 200 unique OTUs in the ND, HFD, H200, H400, and H800 groups, respectively. Significant differences were also found in the ACE, Chao1, Shannon, and Simpson measures of the HFD and H800 groups (Table S3). PCA revealed a significant separation on the microbiota of the groups (Figure 5G). Collectively, these results indicated that HON modulated the gut microbiota of HFD-fed mice. Moreover, the HFD-fed group had significantly reduced Muribaculaceae and Bifidobacterium, and significantly increased Oscillospira and Lactococcus $(P<$ 0.05, Figures 5D,E and Table S4), and decreased Akkermansia 


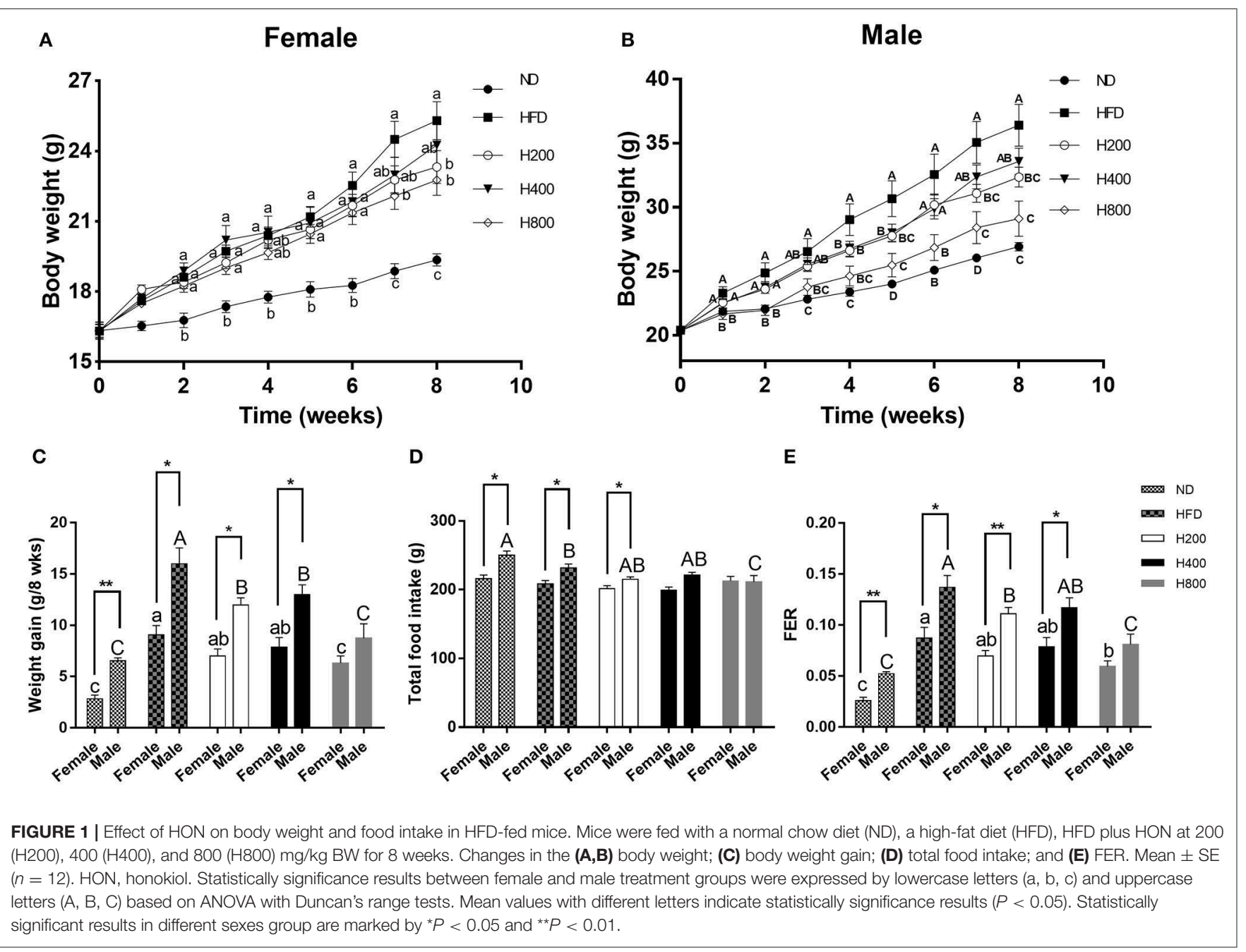

but not significant compared to the ND group (Figures 5D,E and Table S4). The changes in Akkermansia and Oscillospira were opposite to those in the female mice (Figures 5C-F and Table S2). Moreover, compared to the HFD-fed group, the abundances of Akkermansia in all HON treatment, as well as Bacteroides (the SCFA-producing microbiota), Bilophila, Unclassified_Enterobacteriaceae, and Fusobacterium in the H800 group were remarkably enriched. While Muribaculaceae, Oscillospira, and Ruminococcus in all HON treatments, and Unclassified_Clostridiales, Unclassified_Ruminococcaceae, rc44, Lactococcus, and Dehalobacterium in the H800 group were sharply reduced ( $P<0.05$, Figures 5D,H and Table S4). Notably, Akkermansia, which belongs to the phylum Verrucomicrobia, was enriched and Unclassified_Clostridiales, which belongs to the Phylum Fusobacteria, were reduced by HON supplementation in HFD-fed male mice but not female mice.

\section{HON Improves Related Gut Metabolites in HFD-Fed Mice}

Since gut microbiota was modulated by HON supplementation in HFD-fed mice, we next investigated the effect of HON on microbial metabolites, SCFAs, and LBP. The HFD-fed group had a reduced concentration of total SCFAs and increased concentration of LBP in the cecum compared to ND-fed group $(P<0.05$, Figures $6 \mathbf{E}, \mathbf{F})$. HON treatment increased the cecum concentration of SCFAs especially high dose of HON treatment, which markedly increased concentrations of propionate, acetate, butyrate, and total SCFAs, and significantly decreased concentrations of LBP $(P<0.05$, Figures 6 A-C,E,F). Since SCFAs could effectively inhibit the release of LPS endotoxin as shown in Figure 6, HON treatment significantly reduced the concentration of LBP in mice $(P<0.05)$, thereby reducing metabolic endotoxemia in HFD-fed mice, and there were sexrelated in LBP (36).

\section{Correlation of Gut Microbiota With Obesity-Associated Indexes}

Spearman correlation analysis was used to determine the potential correlation between gut microbiota and obesityrelated indexes (Figure 7). There was a significant positive correlation between Dehalobacterium, Oscillospira, rc4-4, Muribaculaceae, and TNF- $\alpha$, LBP, and IL-1 $\beta$ in female mice 


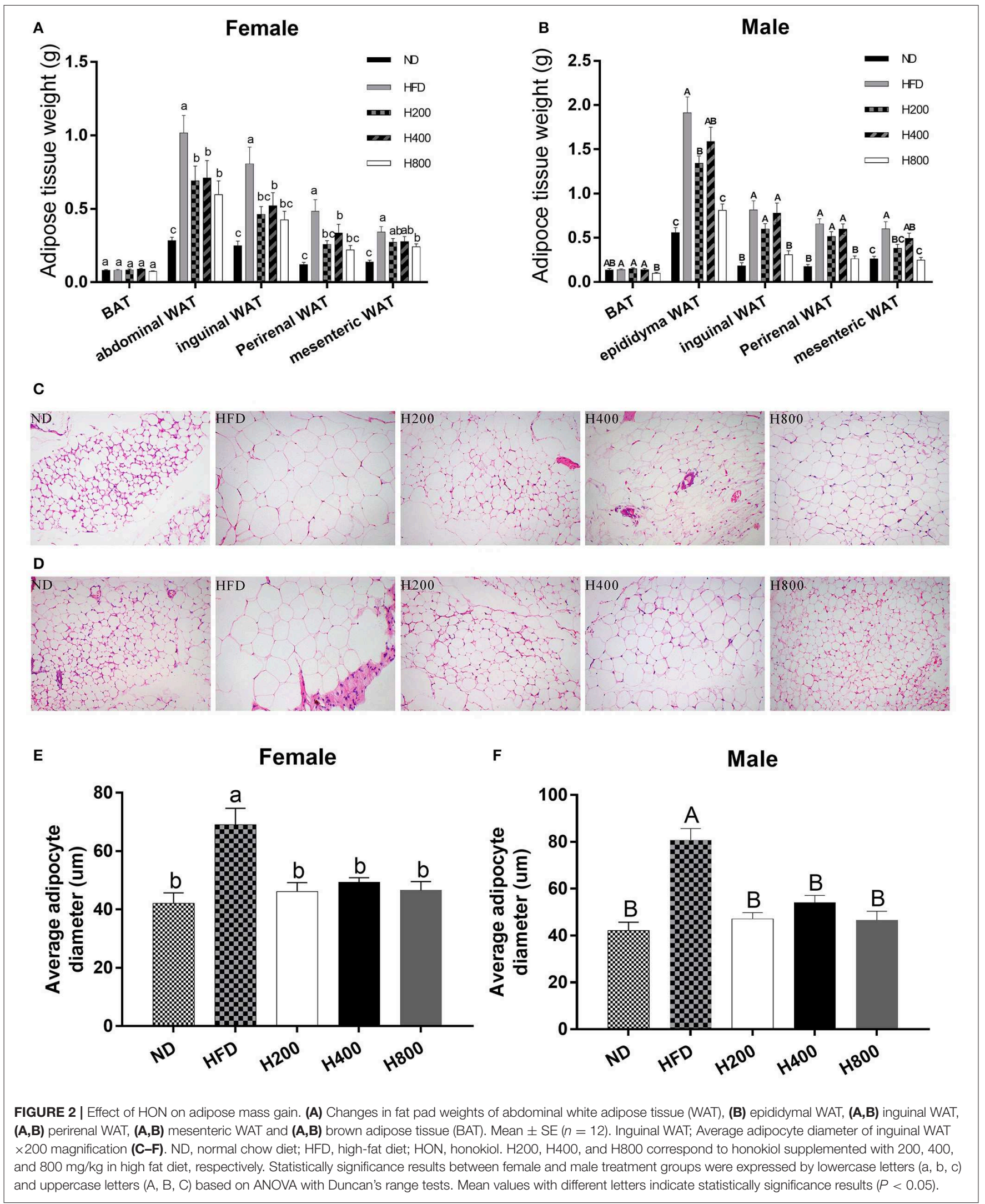




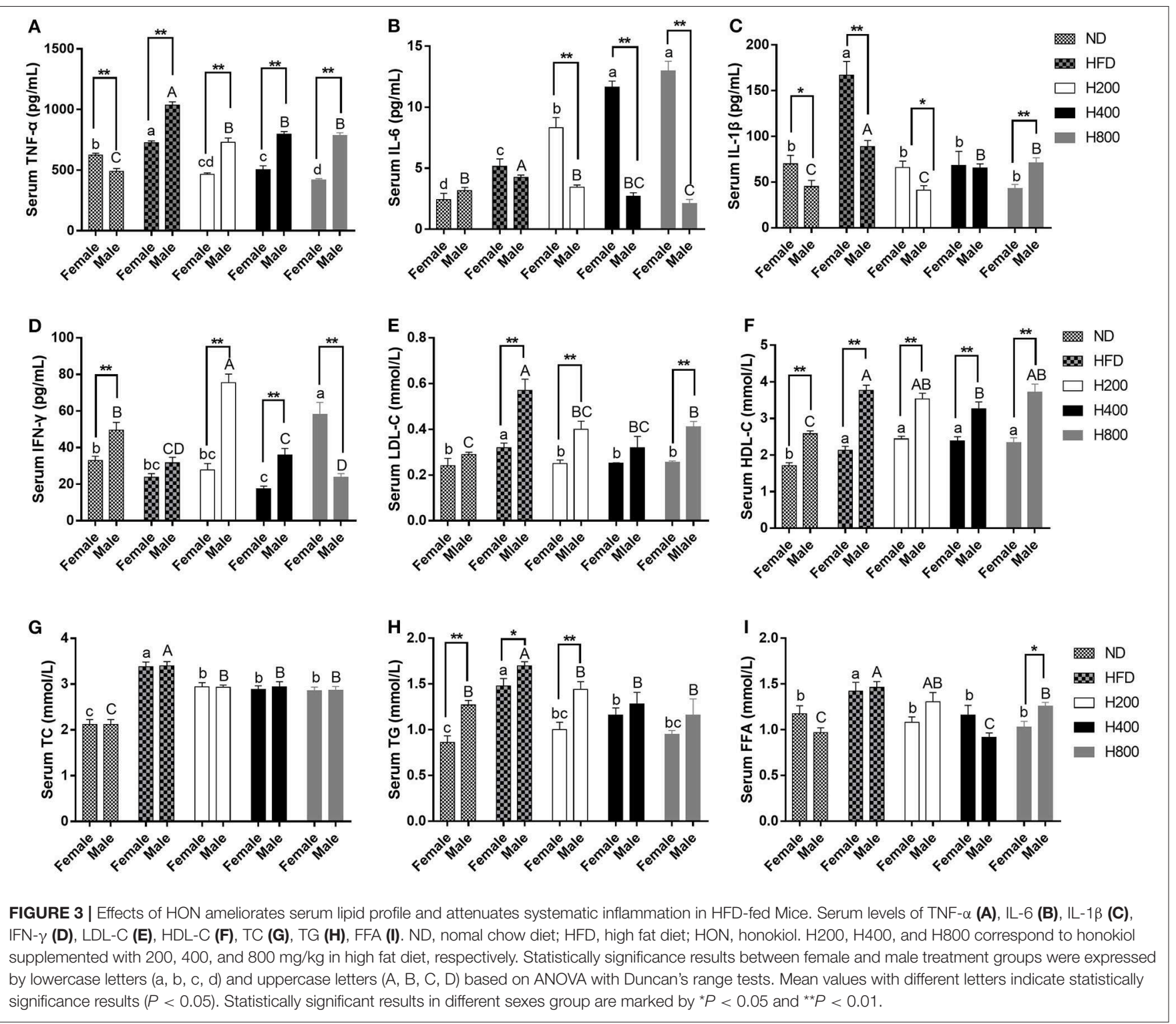

(Figure 7A). Bifidobacterium was negatively correlated with body weight, mesenteric fat and GLU, TG, TC, and HDL-C. Akkermansia was negatively correlated with LBP (Figure 7A). In male mice (Figure 7B), Ruminococcus, Oscillospira Muribaculaceae, Dehalobacterium, Lactobacillus, and rc4-4 showed a significant positive correlation with LBP and IL6, while Unclassified_Enterobacteriaceae were significantly negatively correlated with LBP and IL-6. Bifidobacterium showed a significant negative correlation with body weight, perinenal WAT, inguinal WAT, epididymal WAT, TC, HDLC, LDL-C, HOMA-IR, IL- $1 \beta$, and TNF- $\alpha$, while Lactococcus showed a significant positive correlation with body weight, mesenteric WAT, perinenal WAT, and epididymal WAT, TC, HOMA-IR, TNF- $\alpha$, and IL-6. Notably, as a genus that was differentially regulated by the treatments, Akkermansia was significantly negatively correlated with body weight, perinenal
WAT, inguinal WAT, TG, LBP, and IL-6, and positively correlated with total SCFAs, and propionate. These results suggest that Akkermansia and Bifidobacterium may play an important role in the prevention of obesity and related complications, while Lactococcus may promote the occurrence and development of obesity. Dehalobacterium, Oscillospira, Muribaculaceae, rc4-4, and Ruminococcus may be closely related to the occurrence of obesity. In addition, there were sex-related in the Akkermansia abundance.

\section{DISCUSSION}

Obesity is a common metabolic disease accompanied by numerous health problems (1). As a natural bioactive substance, polyphenols can prevent diet-induced obesity and related 


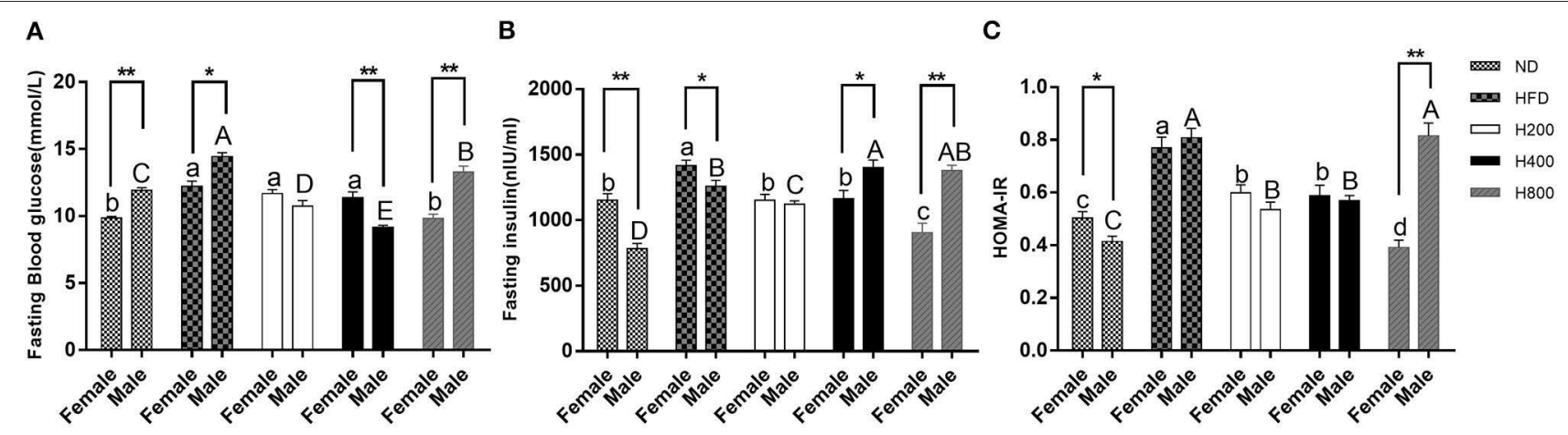

FIGURE 4 | Preventive effects of HON on the development of insulin resistance in HFD-fed mice. Fasting blood glucose (A), fasting insulin (B), and HOMA-IR (C), calculated according to the formula: fasting blood glucose (mmol/L) $\times$ fasting insulin (mIU/L)/22.5 are shown. ND, normal diet; HFD, high-fat diet; HON, honokiol. $\mathrm{H} 2 \mathrm{OO}, \mathrm{H} 400$, and $\mathrm{H} 800$ correspond to honokiol supplemented with 200,400 , and $800 \mathrm{mg} / \mathrm{kg}$ in high fat diet, respectively. Statistically significance results between female and male treatment groups were expressed by lowercase letters ( $a, b, c, d)$ and uppercase letters (A, B, C, D) based on ANOVA with Duncan's range tests. Mean values with different letters indicate statistically significance results $(P<0.05)$. Statistically significant results in different sexes group are marked by ${ }^{\star} P<0.05$ and ${ }^{\star \star} P<0.01$

complications by regulating the composition of gut microbiota $(17,18)$. Previous studies have confirmed that HON can improve diet-induced liver lipid metabolism in obese mice (37, 38). Nevertheless, the effects of it on gut microbiota, and its ability to treat obesity and related complications by regulating the composition of gut microbiota have not previously been reported. In this study, we demonstrated that HON can alleviate obesity and its related complications induced by a HFD by altering gut microbiota, and indicated that sex may be a vital factor affecting the improvement of gut microbiota composition by $\mathrm{HON}$.

A HFD can induce obesity and associated complications in mice, such as excessive fat accumulation, dyslipidemia, inflammation, and increased insulin resistance (39). HON supplementation can reverse the occurrence of high-fat-induced obesity and the above related obesity complications in mice. This suggests that HON has a beneficial effect on obesity, and which is achieved not by lowering food intake, but by reducing the FER in mice. Only a small portion of polyphenols can be absorbed by the small intestine, with most decomposed and utilized by microbiota in the hindgut (24). Recently, a rapidly expanding field of research has shown that the gut microbiota is a significant factor in obesity through the regulation of nutritional access, energy distribution and fat storage (5). Moreover, high fat could bring about an imbalance of gut microbiota in obese people (6) and the gut microbiota has been recognized as a crucial environmental factor in the pathogenesis of obesity (3). These results suggest that polyphenols may improve obesity and its related complications by affecting the gut microbiota.

In addition, when gut microbiota detection was performed on animals with specific genetic background, the results were more evident than those considering different sexes, which was also the reason for inconsistent conclusions in relevant studies $(40,41)$. Therefore, when observing the interaction between the gut microbiota and environmental factors, such as diet, sex as a crucial factor is worth considering $(42,43)$. The evidence indicates that women were less likely to suffer from diet-related metabolic disorders than men $(25,26)$.

Akkermansia is a genus of Gram-negative anaerobic bacteria belonging to the Verrucomicrobia, and its abundance is significantly negatively correlated with obesity. Sustained intervention with a HFD in mice for 8 weeks resulted in a 100 -fold reduction in the abundance of Akkermansia in the cecum (44). The alteration in Akkermansia abundance was correlated with changes in lipid metabolism and the expression of inflammatory response markers (45). Akkermansia intervention could improve obesity and related complications such as fat accumulation, insulin resistance, and circulating levels of inflammatory factors induced by a HFD (44). These results indicate that Akkermansia play an important role in improving obesity and related complications. In addition, it has been demonstrated that polyphenols can improve obesity and related complications by changing the abundance of Akkermansia in the gut (17). In this study, we demonstrated that a HFD resulted in a significant decrease in cecum Akkermansia abundance and that supplementation with $\mathrm{HON}$ led to a significant increase in Akkermansia in male mice. In addition, HON decreased adipose tissue weight and serum inflammatory factor levels, and improved insulin resistance and dyslipidemia in HFD-fed male mice. Correlation analysis also discovered that Akkermansia were negatively correlated with body weight, adipose tissue weight of WAT and TG, LBP, and IL-6, which is consistent with previous studies. These results suggest that HON can improve obesity and related complications by increasing the abundance of Akkermansia in the gut. It is worth noting that the HFD increased the cecum abundance of Akkermansia, and HON supplementation did not have a significant impact on their richness in female mice. This result was contrary to what was seen in male mice, and the correlation analysis of Akkermansia with obesity and related indexes was also different in male and female mice, indicating that Akkermansia abundance may be associated with sex. The study also confirmed that the abundance 
A

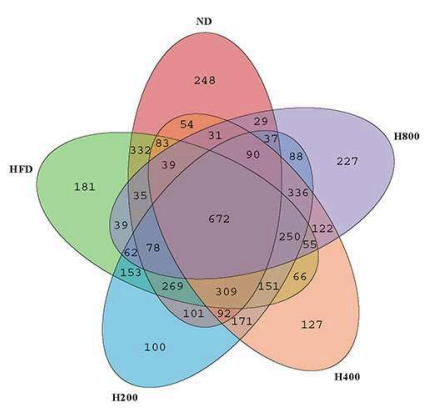

C

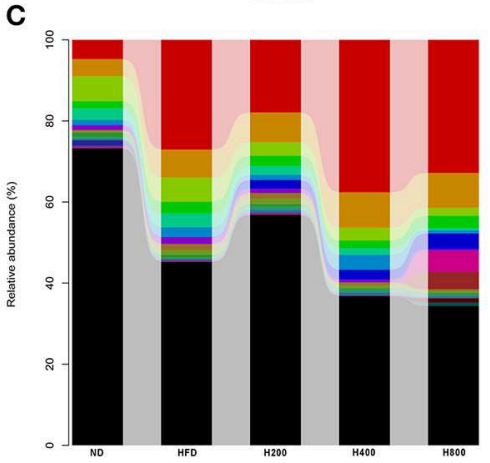

Female

E

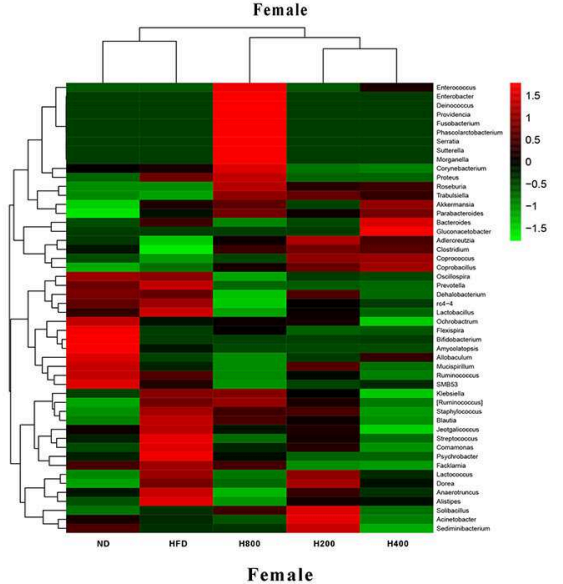

G

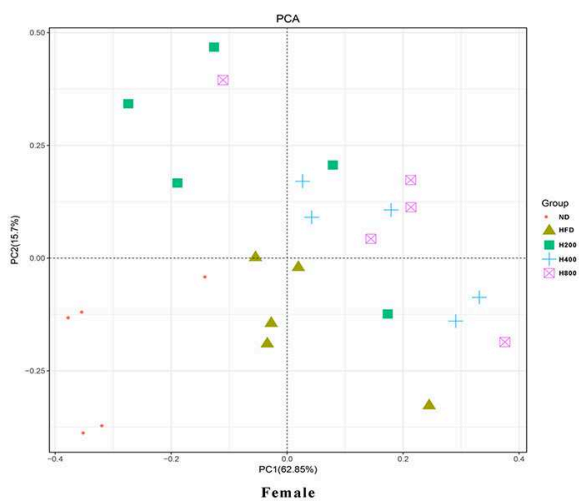

B

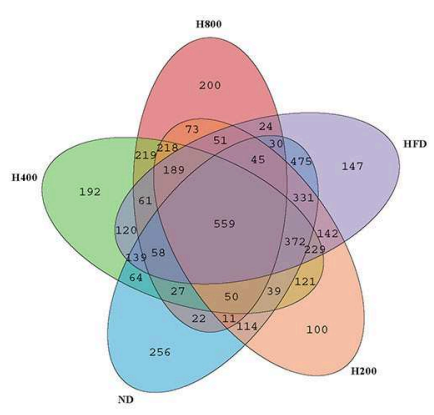

Male

D

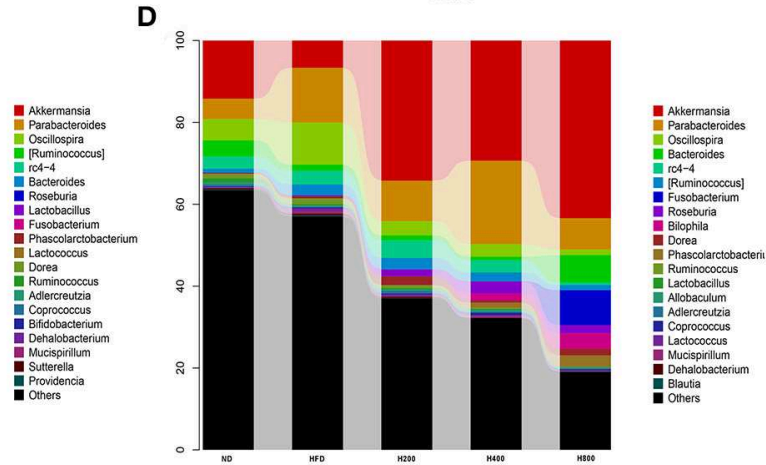

$\mathbf{F}$

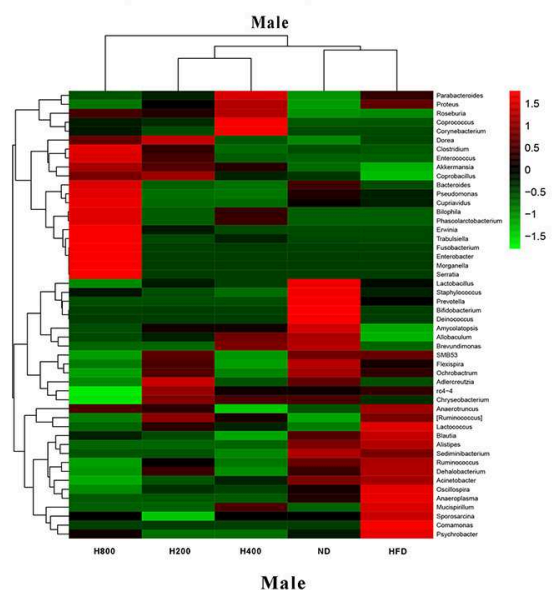

H

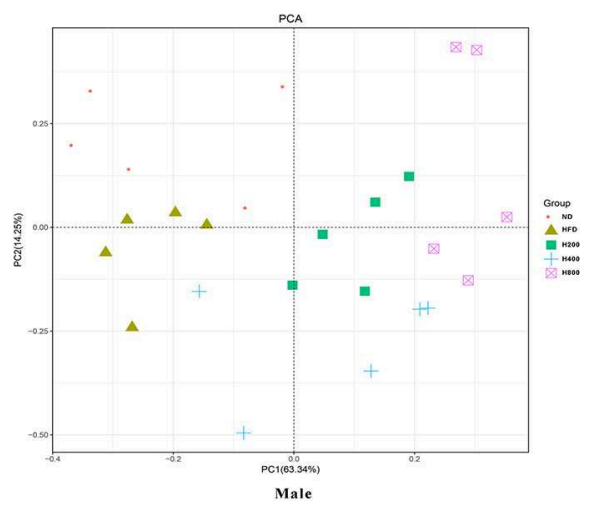

FIGURE 5 | HON alter the composition of the gut microbiota in HFD-fed mice. Venn diagrams showing the unique and shared OTUs in the gut microbiota of the groups (A,B). Community taxonomic composition and abundance distribution map at genus level (C,D). Hierarchically clustered heat map analysis of the top 50 most abundant of gut microbes at genus level (G,H). PCA clustering analysis (E,F). H200, H400, and H800 correspond to honokiol supplemented with 200, 400, and 800 $\mathrm{mg} / \mathrm{kg}$ in high fat diet, respectively. 


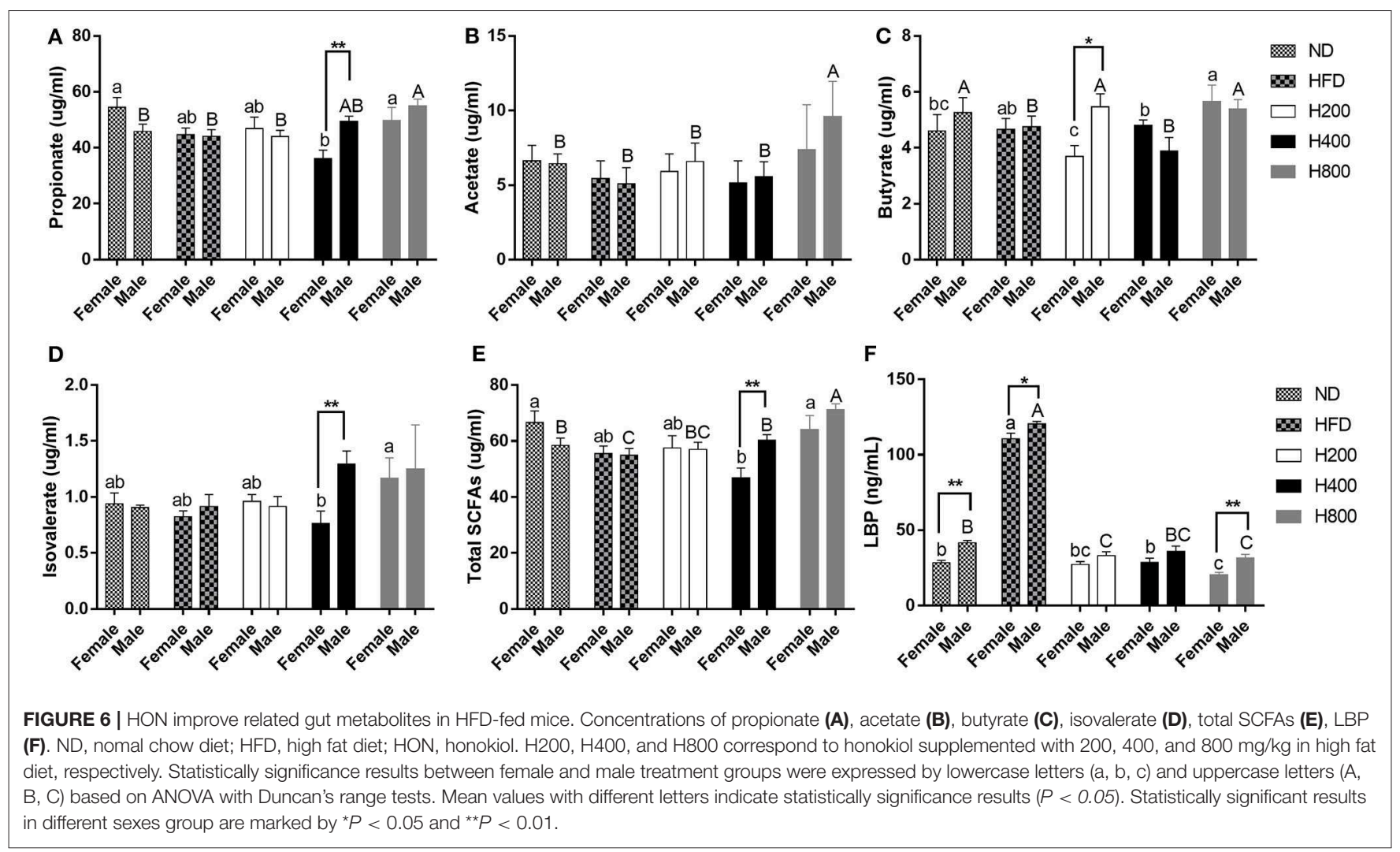

of Akkermansia was varied in mice of different sexes $(43,46)$, which was related to the secretion levels of hormone and bile acid (43).

Bacteroides is a Gram-negative anaerobic belonging to Bacteroidetes, which produce SCFAs. Bacteroides abundance was related to the occurrence and development of obesity and a HFD reduce its abundance $(12,47)$. A Mediterranean diet is associated with more Bacteroides than the Western diet (48). Oral Bacteroides uniformis significantly reduced body weight, increased the number of adipocytes, and improved glucose tolerance, dyslipidemia, and the immune response in obese mice (49). Besides, the abundance of Bacteroides was correlated with sex (50); it decreased with the increasing body mass index (BMI) in men, but there was no correlation in women (50). In this study, we found that the abundance of Bacteroides of male mice in the HFD-fed group was reduced more than 2.5 times, and a high dose of HON significantly increased their abundance of it and the concentration of cecal SCFAs, but there was no significant change in female mice. Correlation analysis also showed that the abundance of Bacteroides was positively correlated with the gut acetate levels in male mice, but there was no such correlation in female mice, which was consistent with the abovementioned results. Moreover, SCFAs can effectively prevent obesity, dyslipidemia, and inflammation (51). Therefore, we speculate that HON may prevent obesity and related complications by producing SCFAs through posterior intestinal fermentation, and that sex is an important factor for different outcomes.
Oscillospira was negatively correlated with emaciation or lower BMI in children and adults $(52,53)$. A Western diet was associated with higher abundance of Oscillospira than a Mediterranean diet (48). A study by Amandine et al. showed that Oscillospira's abundance was positively correlated with obesity index and epididymal adipose tissue (54). A similar result was also found in this study, suggesting that HON may produce anti-obesity effects in HFD-fed mice by reversing the abundance of Oscillospira. In addition, the HFD-fed group of female mice did not have an increased abundance of Oscillospira compared with male mice. Studies also found that Oscillospira's abundance was closely related to sex (42) and that there were sex-based differences in the changes in abundance caused by HFD, which, like Akkermansia, might be related to bile acid and sex hormone secretion (43). Correlation analysis showed that there was a significant positive correlation between Oscillospira and LBP, and TNF- $\alpha$. These findings indicate that Oscillospira may facilitate the occurrence and development of obesity and related complications, and there is a large relationship between gut microbiota and sex.

LBP is an acute phase protein that specifically binds to LPS, promoting innate immunity and thus acts as an ideal biomarker of gut microbiota antigen inflammation. Gut microbiota can reduce serum LPS level (15), and its imbalance can lead to more bacterial lipopolysaccharides entering the bloodstream from the intestinal tract, resulting in inflammatory obesity and insulin resistance in obese mice $(6,48)$. In the present study, the levels of LBP in the 


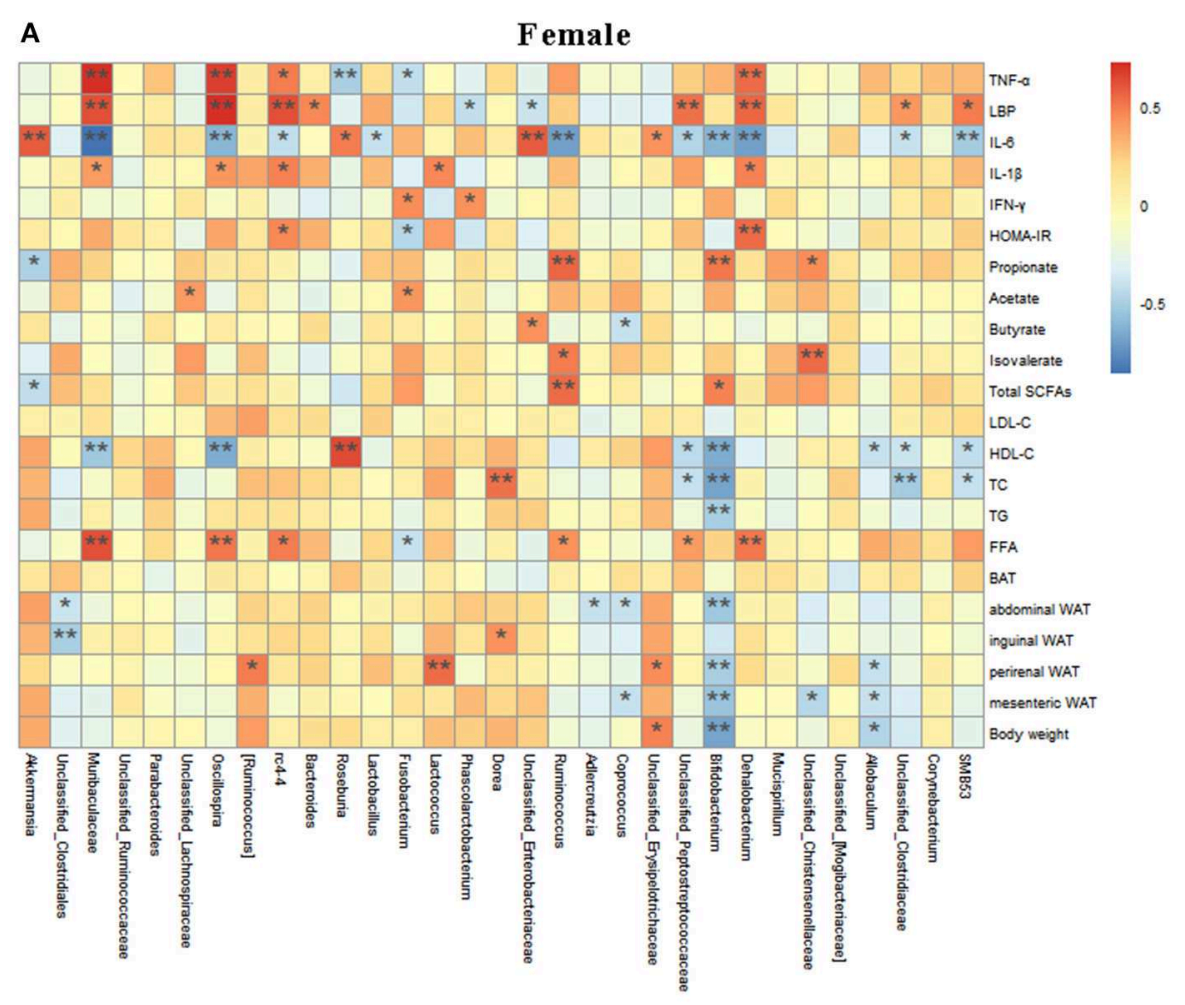

B

Male

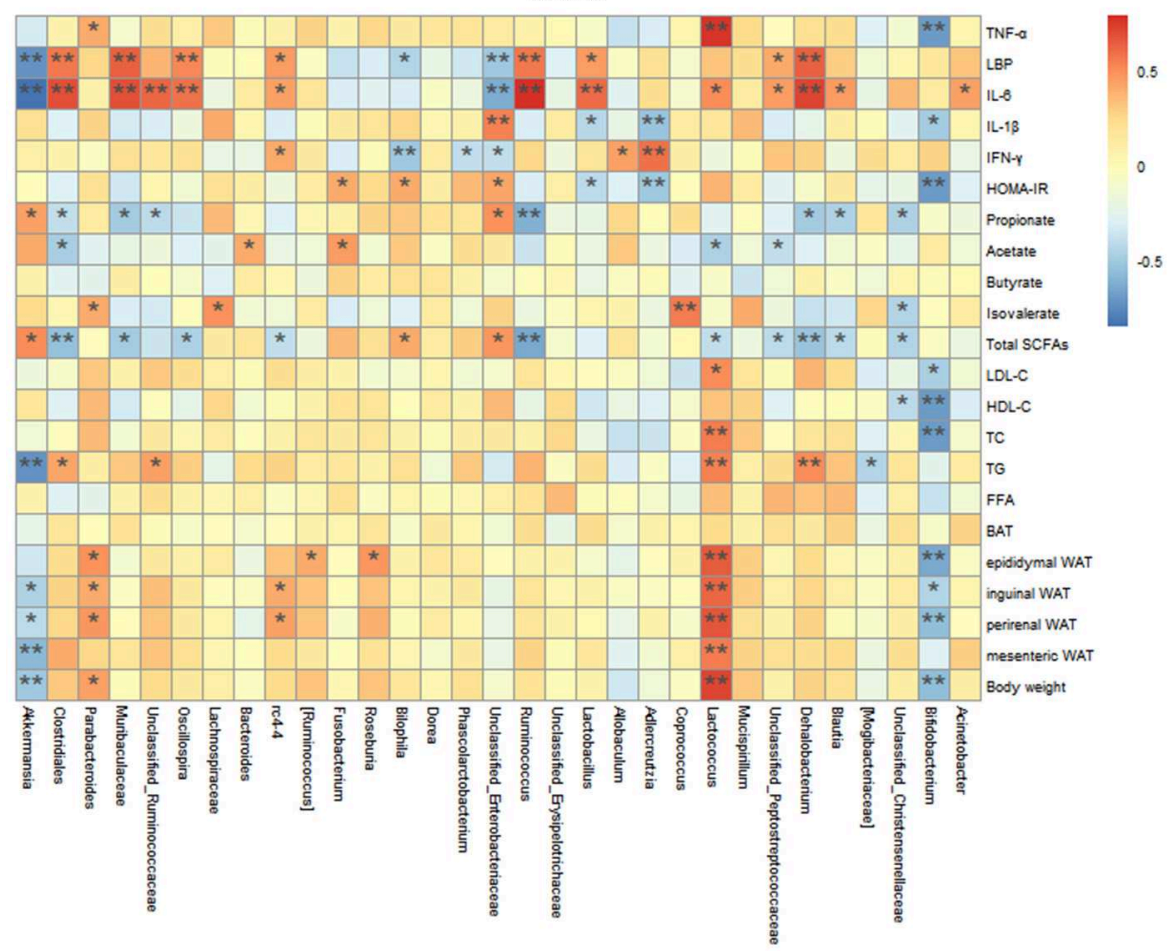

FIGURE 7 | Relationship between intestinal microbiota and Obesity-related Indexes analyzed with a Spelman correlation heatmap. Analysis of correlation between gut microbiota at the genus level and obesity-related indexes are shown in the (A,B). The colors range from blue (negative correlation) to red (positive correlation). ${ }^{*} P<$ 0.05 and ${ }^{\star \star} P<0.01$. 
high-fat group were significantly higher than those in the ND group, and was significantly reduced by adding HON. Correlation analysis showed that there was a significant positive correlation between Dehalobacterium, Oscillospira, Muribaculaceae, rc4-4, and Oscillospira and LBP, which was reduced by supplementation with HON compared to the HFDfed group of mice. This indicates that HON may prevent the occurrence and development of obesity and obesity-related complications by decreasing the amount of LPS produced by gut microbiota.

Surprisingly, in this study, the H800 group had a reduced total food intake and there was no improvement insulin resistance in male mice in this group. We speculated that HON increased the concentration of SCFAs in the cecum, which were absorbed into the hepatic vein system through the intestines, promoted liver metabolism processes, such as gluconeogenesis, provided energy for liver metabolism (55). Elegant rodent studies have demonstrated that SCFAs have a suppressive effect on appetite and energy intake via central nervous system-related mechanisms and the gut-brain axis (56, 57). In addition, HON may have dose-dependent effects on gut microbiota. This study found that compared with other doses, the high dose of $\mathrm{HON}$ significantly reduced the richness of the gut microbiota, which also reduced the abundance of Unclassified_Clostridiales significantly in male mice, and reduced the abundance of Muribaculaceae more significantly in female mice.

\section{CONCLUSIONS}

HON can prevent diet-induced obesity and associated complications by regulating gut microbiota and metabolites. Sex and dose may be important factors that deserve our attention while using this compound. This study provides a new perspective on the anti-obesity effect of the Chinese medicinal

\section{REFERENCES}

1. Abarca-Gómez L, Abdeen ZA, Hamid ZA, Abu-Rmeileh NM, Acosta-Cazares B, Acuin C, et al. Worldwide trends in body-mass index, underweight, overweight, and obesity from 1975 to 2016: a pooled analysis of 2416 population-based measurement studies in 128.9 million children, adolescents, and adults. Lancet. (2017) 390:2627-42. doi: 10.1016/S0140-6736(17)32129-3

2. Carmody R, Gerber GK, Luevano JM Jr, Gatti D, Somes L, Svenson K, et al. Diet dominates host genotype in shaping the murine gut microbiota. Cell Host Microbe. (2015) 17:72-84. doi: 10.1016/j.chom.2014.11.010

3. Bäckhed F, Ding H, Wang T, Hooper LV, Koh GY, Nagy A, et al. The gut microbiota as an environmental factor that regulates fat storage. Proc Natl Acad Sci USA. (2004) 101:15718-23. doi: 10.1073/pnas.0407076101

4. Turnbaugh PJ, Ley RE, Mahowald MA, Vincent M, Mardis ER, Gordon JI. An obesity-associated gut microbiome with increased capacity for energy harvest. Nature. (2006) 444:1027-31. doi: 10.1038/nature05414

5. Zhang C, Zhang M, Wang S, Han R, Cao Y, Hua W, et al. Interactions between gut microbiota, host genetics and diet relevant to development of metabolic syndromes in mice. ISME J. (2010) 4:232-41. doi: 10.1038/ismej.2009.112

6. Shen J, Obin MS, Zhao L. The gut microbiota, obesity and insulin resistance. Mol Aspects Med. (2013) 34:39-58. doi: 10.1016/j.mam.2012.11.001 herbal extract $\mathrm{HON}$, but its precise mechanism of action needs to be further verified.

\section{DATA AVAILABILITY STATEMENT}

The datasets generated for this study can be found in the NCBI - SRR10432877.

\section{ETHICS STATEMENT}

The animal study was reviewed and approved by Ethics of Animal Experiments of Hunan Agriculture University.

\section{AUTHOR CONTRIBUTIONS}

$\mathrm{YD}, \mathrm{ZF}, \mathrm{XH}$, and $\mathrm{ZS}$ designed the experiment and revised the manuscript. YD, HL, LC, and TP carried out the animal trials and sample analysis. YD and XG did some data analysis work and editor of the paper. $\mathrm{ZF}, \mathrm{XH}$, and $\mathrm{ZS}$ is responsible for the integrity of the work as a whole. All authors reviewed and approved the final manuscript.

\section{FUNDING}

This work was supported by the National Key R\&D Program of Intergovernmental Key Projects (2018YFE0101700), National Natural Science Foundation of China (31872378), and the Double first-class construction project of Hunan Agricultural University (SYL201802015, SYL201802009).

\section{SUPPLEMENTARY MATERIAL}

The Supplementary Material for this article can be found online at: https://www.frontiersin.org/articles/10.3389/fimmu. 2019.02800/full\#supplementary-material

7. Emmanuelle LC, Trine N, Junjie Q, Edi P, Falk H, Gwen F, et al. Richness of human gut microbiome correlates with metabolic markers. Nature. (2013) 500:541-6.

8. Turnbaugh PJ, Bäckhed F, Fulton L, Gordon JI. Diet-Induced obesity is linked to marked but reversible alterations in the mouse distal gut microbiome. Cell Host Microbe. (2008) 3:213-23. doi: 10.1016/j.chom.2008.02.015

9. Bäckhed F, Manchester JK, Semenkovich CF, Gordon JI. Mechanisms underlying the resistance to diet-induced obesity in germ-free mice. Proc Natl Acad Sci USA. (2007) 104:979-84. doi: 10.1073/pnas.0605374104

10. Na-Ri S, June-Chul L, Hae-Youn L, Min-Soo K, Tae Woong W, Myung-Shik L, et al. An increase in the Akkermansia spp. population induced by metformin treatment improves glucose homeostasis in diet-induced obese mice. Gut. (2014) 63:727-35. doi: 10.1136/gutjnl-2012-303839

11. Amandine E, Clara B, Lucie G, Ouwerkerk JP, Céline D, Bindels LB, et al. Cross-talk between Akkermansia muciniphila and intestinal epithelium controls diet-induced obesity. Proc Natl Acad Sci USA. (2013) 110:906671. doi: 10.1073/pnas.1219451110

12. Cani PD, Neyrinck AM, Fava F, Knauf C, Burcelin RG, Tuohy KM, et al. Selective increases of bifidobacteria in gut microflora improve high-fat-dietinduced diabetes in mice through a mechanism associated with endotoxaemia. Diabetologia. (2007) 50:2374-83. doi: 10.1007/s00125-007-0791-0 
13. Santisteban MM, Qi Y, Zubcevic J, Kim S, Yang T, Shenoy V, et al. Hypertension-linked pathophysiological alterations in the gut. Circ Res. (2016) 120:312-23. doi: 10.1161/CIRCRESAHA.116.309006

14. Cani PD, Jacques A, Miguel Angel I, Marjorie P, Claude K, Delphine B, et al. Metabolic endotoxemia initiates obesity and insulin resistance. Diabetes. (2007) 56:1761-72. doi: 10.2337/db07-1181

15. Cani PD, Bibiloni R, Knauf C, Waget A, Neyrinck AM, Delzenne NM, et al. Changes in gut microbiota control metabolic endotoxemia-induced inflammation in high-fat diet-induced obesity and diabetes in mice. Diabetes. (2008) 57:1470-81. doi: 10.2337/db07-1403

16. Belkaid Y, Hand T. Role of the microbiota in immunity and inflammation. Cell. (2014) 157:121-41. doi: 10.1016/j.cell.2014.03.011

17. Masumoto S, Terao A, Yamamoto Y, Mukai T, Miura T, Shoji T. Nonabsorbable apple procyanidins prevent obesity associated with gut microbial and metabolomic changes. Sci Rep. (2016) 6:31208. doi: 10.1038/srep31208

18. Wang $\mathrm{P}, \mathrm{Li} \mathrm{D}, \mathrm{Ke} \mathrm{W}$, Liang $\mathrm{D}, \mathrm{Hu} \mathrm{X}$, Chen F. Resveratrol-induced gut microbiota reduces obesity in high-fat diet-fed mice. Int $J$ Obes. (2019). doi: 10.1038/s41366-019-0332-1. [Epub ahead of print].

19. Woodbury A, Yu SP, Wei L, García P. Neuro-modulating effects of honokiol: a review. Front Neurol. (2013) 4:130. doi: 10.3389/fneur.2013.00130

20. Yongjin HU, Qiao J, Zhang XI, Changrong GE. Antimicrobial activity of Magnolia officinalis extracts in vitro and its effects on the preservation of chilled mutton. J Food Biochem. (2011) 35:425-41. doi: 10.1111/j.1745-4514.2010.00393.x

21. Konoshima T, Kozuka M, Tokuda H, Nishino H, Iwashima A, Haruna M, et al. Studies on inhibitors of skin tumor promotion, IX. Neolignans from Magnolia officinalis. J Nat Prod. (1991) 54:816-22. doi: 10.1021/np50075a010

22. Zhao C, Liu ZQ. Comparison of antioxidant abilities of magnolol and honokiol to scavenge radicals and to protect DNA. Biochimie. (2011) 93:175560. doi: 10.1016/j.biochi.2011.06.012

23. Kim YJ, Choi MS, Cha BY, Woo JT, Park YB, Kim SR, et al. Longterm supplementation of honokiol and magnolol ameliorates body fat accumulation, insulin resistance, and adipose inflammation in high-fat fed mice. Mol Nutr Food Res. (2013) 57:1988-98. doi: 10.1002/mnfr.201300113

24. Stevens JF, Maier CS. The chemistry of gut microbial metabolism of polyphenols. Phyochem Rev. (2016) 15:42544. doi: 10.1007/s11101-016-9459-z

25. Priego T, Sánchez J, Picó C, Palou A. Sex-associated differences in the leptin and ghrelin systems related with the induction of hyperphagia under high-fat diet exposure in rats. Hormones Behav. (2009) 55:3340. doi: 10.1016/j.yhbeh.2008.07.010

26. Medrikova D, Jilkova ZM, Bardova K, Janovska P, Rossmeisl M, Kopecky J. Sex differences during the course of diet-induced obesity in mice: adipose tissue expandability and glycemic control. Int J Obes. (2012) 36:26272. doi: $10.1038 /$ ijo. 2011.87

27. Hold GL, Smith M, Grange C, Watt ER, El-Omar EM, Mukhopadhya I. Role of the gut microbiota in inflammatory bowel disease pathogenesis: what have we learnt in the past 10 years? World J Gastroenterol. (2014) 20:1192-210. doi: 10.3748/wjg.v20.i5.1192

28. Molodecky NA, Ing Shian S, Rabi DM, Ghali WA, Mollie F, Greg C, et al. Increasing incidence and prevalence of the inflammatory bowel diseases with time, based on systematic review. Gastroenterology. (2011) 142:4654. doi: 10.1053/j.gastro.2011.10.001

29. Chen H, Jiang W. Application of high-throughput sequencing in understanding human oral microbiome related with health and disease. Front Microbiol. (2014) 5:508. doi: 10.3389/fmicb.2014.00508

30. Edgar RC. Search and clustering orders of magnitude faster than BLAST. Bioinformatics. (2010) 26:2460-1. doi: 10.1093/bioinformatics/btq461

31. Lozupone CA, Micah H, Kelley ST, Rob K. Quantitative and qualitative beta diversity measures lead to different insights into factors that structure microbial communities. Appl Environ Microbiol. (2007) 73:157685. doi: 10.1128/AEM.01996-06

32. Alban R. Multivariate analyses in microbial ecology. Fems Microbiol Ecol. (2010) 62:142-60. doi: 10.1111/j.1574-6941.2007.00375.x

33. Zaura E, Keijser BJ, Huse SM, Crielaard W. Defining the healthy "core microbiome" of oral microbial communities. BMC Microbiol. (2009) 9:259. doi: 10.1186/1471-2180-9-259
34. Shang Q, Song G, Zhang M, Shi J, Xu C, Hao J, et al. Dietary fucoidan improves metabolic syndrome in association with increased Akkermansia population in the gut microbiota of high-fat diet-fed mice. J Funct Foods. (2017) 28:138-46. doi: 10.1016/j.jff.2016.11.002

35. Ley RE, Fredrik BC, Peter T, Lozupone CA, Knight RD, Gordon JI. Obesity alters gut microbial ecology. Proc Natl Acad Sci USA. (2005) 102:110705. doi: $10.1073 /$ pnas. 0504978102

36. Liu Z, Chen Z, Guo H, He D, Zhao H, Wang Z, et al. The modulatory effect of infusions of green tea, oolong tea, and black tea on gut microbiota in high-fatinduced obese mice. Food Funct. (2016) 7:4869-79. doi: 10.1039/C6FO01439A

37. Suk SM, Jung Hwan K, Hye Jung K, Ki Churl C, Won PS. Honokiol activates the LKB1-AMPK signaling pathway and attenuates the lipid accumulation in hepatocytes. Toxicol Appl Pharmacol. (2015) 284:11324. doi: 10.1016/j.taap.2015.02.020

38. Lee JH, Jung JY, Jang EJ, Jegal KH, Moon SY, Ku SK, et al. Combination of honokiol and magnolol inhibits hepatic steatosis through AMPK-SREBP-1c pathway. Exp Biol Med. (2015) 240:508-18. doi: 10.1177/1535370214547123

39. Heymsfield SB, Wadden TA. Mechanisms, pathophysiology, and management of obesity. N Engl J Med. (2017) 376:1490-1. doi: 10.1056/NEJMc1701944

40. Christophe L, Lionel RG, Kim HM, Mirjana R, Vaughan EE, Vos WMD, et al. Colonic microbiota signatures across five northern European countries. Appl Environ Microbiol. (2005) 71:4153. doi: 10.1128/AEM.71.7.4153-4155.2005

41. Huttenhower C, Gevers D, Knight R, Abubucker S, Badger JH, Chinwalla AT, et al. Structure, function and diversity of the healthy human microbiome. Nature. (2012) 486:207-14. doi: 10.1038/nature11234

42. Bolnick DI, Snowberg LK, Hirsch PE, Lauber CL, Org E, Parks B, et al. Individual diet has sex-dependent effects on vertebrate gut microbiota. Nat Commun. (2015) 5:4500. doi: 10.1038/ncomms5500

43. Org E, Mehrabian M, Parks BW, Shipkova P, Liu X, Drake TA, et al. Sex differences and hormonal effects on gut microbiota composition in mice. Gut Microbes. (2016) 7:313-22. doi: 10.1080/19490976.2016.1203502

44. Markle JGM, Frank DN, Mortin-Toth S, Robertson CE, Feazel LM, Rolle-Kampczyk U, et al. Sex differences in the gut microbiome drive hormone-dependent regulation of autoimmunity. Science. (2013) 339:10848. doi: 10.1126/science.1233521

45. Schneeberger M, Everard A, Gómez-Valadés AG, Matamoros S, Ramírez $\mathrm{S}$, Delzenne NM, et al. Akkermansia muciniphilainversely correlates with the onset of inflammation, altered adipose tissue metabolism and metabolic disorders during obesity in mice. Sci Rep. (2015) 5:16643. doi: $10.1038 /$ srep 16643

46. Coretti L, Cristiano C, Florio E, Scala G, Lama A, Keller S, et al. Sex-related alterations of gut microbiota composition in the BTBR mouse model of autism spectrum disorder. Sci Rep. (2017) 7:45356. doi: 10.1038/srep45356

47. Yadav H. Gut microbiome composition in non-human primates consuming a western or Mediterranean diet. Front Nutr. (2018) 5:28. doi: 10.3389/fnut.2018.00028

48. Zhu Z, Zhu B, Sun Y, Ai C, Wang L, Wen C, et al. Sulfated polysaccharide from sea cucumber and its depolymerized derivative prevent obesity in association with modification of gut microbiota in high-fat diet-fed mice. Mol Nutr Food Res. (2018) 62:e1800446. doi: 10.1002/mnfr.201800446

49. Gauffin CP, Santacruz A, Moya Á, Sanz Y. Bacteroides uniformis CECT 7771 ameliorates metabolic and immunological dysfunction in mice with high-fat-diet induced obesity. PloS ONE. (2012) 7:e41079. doi: 10.1371/journal.pone.0041079

50. Haro C, Rangel-Zúñiga OA, Alcalá-Díaz JF, Gómez-Delgado F, Pérez-Martínez P, Delgado-Lista J, et al. Intestinal microbiota is influenced by gender and body mass index. PloS ONE. (2016) 11:e154090. doi: 10.1371/journal.pone.0154090

51. Lu Y, Fan C, Li P, Lu Y, Chang X, Qi K. Short chain fatty acids prevent highfat-diet-induced obesity in mice by regulating $\mathrm{G}$ protein-coupled receptors and gut microbiota. Sci Rep. (2016) 6:37589. doi: 10.1038/srep37589

52. Tims S, Derom C, Jonkers DM, Vlietinck R, Saris WH, Kleerebezem M, et al. Microbiota conservation and BMI signatures in adult monozygotic twins. ISME J. (2013) 7:707-17. doi: 10.1038/ismej.2012.146

53. Goodrich JK, Waters JL, Poole AC, Sutter JL, Omry K, Ran B, et al. Human genetics shape the gut microbiome. Cell. (2014) 159:78999. doi: $10.1016 /$ j.cell.2014.09.053 
54. Amandine E, Sébastien M, Lucie G, Delzenne NM, Cani PD. Saccharomyces boulardii administration changes gut microbiota and reduces hepatic steatosis, low-grade inflammation, and fat mass in obese and type 2 diabetic db/db mice. Mbio. (2014) 5:e1011-14. doi: 10.1128/mBio. 01011-14

55. Brüssow H, Parkinson SJ. You are what you eat. Nat Biotechnol. (2014) 32:243-5. doi: 10.1038/nbt.2845

56. De Vadder F, Kovatcheva-Datchary P, Goncalves D, Vinera J, Zitoun C, Duchampt A, et al. Microbiota-generated metabolites promote metabolic benefits via gut-brain neural circuits. Cell. (2014) 156:84-96. doi: 10.1016/j.cell.2013. 12.016

57. Frost G, Sleeth ML, Sahuri-Arisoylu M, Lizarbe B, Cerdan S, Brody $\mathrm{L}$, et al. The short-chain fatty acid acetate reduces appetite via a central homeostatic mechanism. Nat Commun. (2014) 5:3611. doi: 10.1038/ ncomms 4611

Conflict of Interest: The authors declare that the research was conducted in the absence of any commercial or financial relationships that could be construed as a potential conflict of interest.

Copyright (C) 2019 Ding, Song, Li, Chang, Pan, Gu, He and Fan. This is an open-access article distributed under the terms of the Creative Commons Attribution License (CC $B Y)$. The use, distribution or reproduction in other forums is permitted, provided the original author(s) and the copyright owner(s) are credited and that the original publication in this journal is cited, in accordance with accepted academic practice. No use, distribution or reproduction is permitted which does not comply with these terms. 